\title{
A Survey on Denoising Methods
}

\author{
Rosebell John ${ }^{1}$, V. Karunakaran ${ }^{2}$ \\ ${ }^{1}$ Post-Graduate Student, Department of Computer Science and Engineering, Karunya University, India \\ ${ }^{2}$ Assistant professor, Department of Computer Science and Engineering, Karunya University, India
}

\begin{abstract}
The impulse is present in the image, this will hide the essential details of the image and decrease the clearness of the image. There for the denoising is very important. This paper reviewed on different types of denoising methods. These denoising methods are compared with each other using PSNR value and the efficiency of these methods is calculated.
\end{abstract}

Keywords: Corrupted pixel, Denoising, Filtering, Median filter, Noise detection.

\section{Introduction}

Noise is undesirable signal. Noise is the effect of errors in the image achievement method that result in pixel values that do not replicate the true intensities of the actual picture. The noise in the image will degrade the quality of the image and loose the information in image There are number of ways that noise can be introduce into an image. For example: The image is taken from a photograph made on film, if the image is acquired directly in a digital format, the instrument for meeting the data can establish the noise, electronic transmission of image data can set up noise. There are many types of noises are available: Gaussian noise, impulse noise, uniform noise and shot noise. Gaussian noise is a noise available in the image, here each pixel affected by the noise. The case of impulse noise, salt and pepper is the example of impulse noise. This type of noise is affecting a small amount of image pixels. When viewed, the image contains both dark and white dots.

The removal of noise from the image is known as denoising. Image denoising is one of the most fundamental, broadly studied, and unsolved problems in image processing, the flow of denoising is displayed in Fig.1.The goal of noise removal is to suppress the noise while saving the integrity of edge and detail information in the image. Noise removal is classified into two types: linear and non linear filtering. In linear filtering denoising techniques is directly applied to the image pixel without checking the availability of noisy and non noisy pixels.

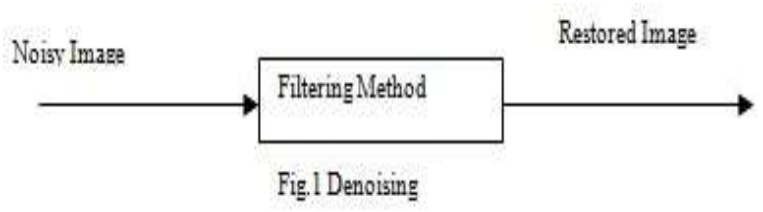

The examples of linear filtering are Mean Filter [1]. The disadvantage of this filter is it will affect the quality of non noisy pixel. In the case of non linear filter, this is done by two steps first detection then filtering. First step the position of the noise is detected and in the second step replace the noisy pixel with calculated value. The example of nonlinear filter is Median Filter [2],Weighted Median Filter (WMF) [3], Center Weighted Median Filter (CWMF) [4], Adaptive Median Filter(AMF) [5], Progressive Switching Median Filter (ASMF) [7], Tri-state Median Filter (TMF) [6], Rank-order based Adaptive Median Filter (RAMF) [9], Minmax Detector Based (MDB) filter [10], Adaptive Rank Order Filter [8], Robust Estimation Algorithm (REA) [11], Decision Based Adaptive Median Filter [12].

\section{Denoising techniques}

\subsection{Mean Filter}

Mean filtering is usually used as a simple method for reducing noise in an image. Mean filtering is a simple, spontaneous and easy to execute method of smoothing images [1]. The calculated median value is replaced with center value. 


\subsection{Median Filter}

It provides better removal of impulse noise from corrupted images. It replaces the center value with the median of all the pixels in the window. According to window size each pixels in the window is taken and sort the pixel then find the median of the each window. Then this median value is replaced by center value.

\subsection{Weighted Median Filter}

The basic idea is to give weight to the each pixel. Every pixel is given a weight. This weight is multiply with pixel. According to this weight the pixels are sort into ascending order, and then find the median value from the sorted list. This value is replaced with center value.

\subsection{Center Weighted Median Filter}

In CWM center pixel of window is considered as test pixel. Check that the center pixel is less than minimum value available in window and center pixel is greater than maximum value available in window then center pixel is considered as corrupted pixel. This corrupted pixel is replaced by calculated value. Weight is given to the center pixel then sorts all element of window in ascending order and calculate median of elements.

\subsection{Tri-state Median Filter}

This will work according to the threshold value. First take each pixel from the window then check that this pixel is less than the predefined threshold value, and then this pixel is consider as uncorrupted pixel and keep the pixel as it is. Otherwise calculate Center Weighted Median (CWM) filter value and Standard Median (SM) value. Check that the threshold is placed between CWM and SM, and then the corrupted pixel is replaced with CWM value. If the SM is greater than threshold then the SM value is placed instead of noisy pixel. The range of threshold is 0 to 255 .

\subsection{Adaptive Median Filter}

It uses changing window size to denoising. The window size is increases until get the correct value for median is calculated and noise pixel is replaced with calculated median value. Here two conditions are used one to find corrupted pixels and second one is to check accuracy of median value. Check that the pixel is less than minimum value available in window and also the pixel is greater than maximum value present in window then center pixel is considered as corrupted pixel. Then check that the median value is less than minimum pixel value available in window and median is greater than maximum pixel value available in window, then median value is judge as noisy pixel. If the median is noisy one then increase the window size and again calculate the median value of the pixel until get correct median value. While increasing the window size it will consider the previous median value also.

\subsection{Progressive Switching Median Filter}

Here one pixel is taken for test pixel from the window. Check that the pixel is less than the minimum value of the pixel available in the window and greater than the maximum value of the pixel available in the window then central pixel is considered as noisy pixel. Second noise pixels are replaced by estimated median value. Then check that the median value is less than minimum pixel value available in window and greater than maximum pixel value available in window, then median value is consider as noisy pixel. If the median is noisy one then increase the window size and again calculate the median value of the pixel until get correct median value. Here median is calculated without considering the noisy pixel available in window. Then check that the calculated median value is less than minimum pixel available in window and greater than maximum pixel available in window then median value is consider as corrupted value. If the median is noisy one then increase the window size and again calculate the median value of the pixel until get correct median value.

\subsection{Rank-Order Based Adaptive Median Filter}

In RAMF, a pixel is taken if the median of a window is placed between the minimum and the maximum value of the window and the pixel is exactingly between the minimum and the maximum value of the window; In case, the median of the window under concern is exactingly between the minimum and the maximum value of the window and the pixel does not lie between the minimum and the maximum value of the window, then the pixel is replaced by the median value; otherwise, the size of the window is increased and the pixel will be replaced by the median of the increased size window, also the new median is between the minimum and the maximum value of the window, otherwise, the window size is again increased up to pre-fixed maximum level, otherwise the central pixel is keep unchanged. 


\subsection{Minmax Detector Based filter}

The goal of this method is to restructure the true image from the corrupted image. MDB should work at high noise image and superior to other existing techniques. Here the center pixel is taken as tested pixel. Then check that the test pixel is less than minimum value present in the window or test pixel is greater than the maximum value present in the window then test pixel is corrupted. If the test pixel is corrupted then find the median value apply that value to the corrupted pixel. Shift the window row wise then column wise to cover all the pixels in the image, and then continue the above checking.

\subsection{Adaptive Rank Order Filter}

Here check that center pixel is noisy or not. If yes then sort the pixels in the window in ascending order. Then find the minimum, maximum and median value. Then check that the median value is noisy one or not by checking the median is placed between min and max value, if yes med is not a noisy pixel then replace center value with median value. Otherwise expand the window size. Repeat the steps until all pixels are process.

\subsection{Robust Estimation algorithm}

First find the median, and then find the minimum value and maximum value from the selected window. Check that minimum value is less than median value and the median value is less than maximum value from the window. If the condition is true, then check that the test pixel is placed between the minimum and maximum value if it is trues then that pixel is not a noisy one. Otherwise find absolute deviation of the each pixel [11]. If the absolute deviation is zero neighbourhood pixel is consider as corrupted one, else calculate robust influence function using this calculated value the pixel is calculate.

\subsection{Decision Based Adaptive Median Filter}

Here two stages are to remove the noise. In the first stage noise pixel is detect using rank order absolute difference. In the second stage noisy pixel is replaced by calculated median value. Find the absolute deviation for the entire pixel. Then sort the calculated absolute deviation and find the sum of the smallest absolute deviations are calculated, this is called ROAD [12]. The ROAD value is compare with threshold. Based on this comparison the noisy pixel is identified. In the case of filtering, check that flag in the binary image is zero then that pixel is not noisy one, otherwise check that number of non zero pixels are three or above then the pixel is replaced with median of non zero pixels. If the number of nonzero pixel is less than three then increase the initial value and do the steps from the beginning.

\subsection{Robust Outlyingness Ratio-Nonlocal Mean filter}

This case the denoising consists of two steps, detection followed by filtering. The detection contains two stages coarse and fine stage. Here the steps are same in the both stages only difference is that, in the case of coarse stage large threshold is given through this the false-hit term become small and miss term become large [13]. The output of coarse stage is given to fine stage, here in the fine stage the threshold is taken as small then the false-hit become large and miss term become small. In the detection first calculate the ROR value for each pixel then according to ROR value the pixel is divided into four clusters. Then find the absolute deviation, then compare the absolute deviation with threshold according to this comparison the noise pixel is identified. The result is added to the flag detection matrix. This detection flag matrix is given to nonlocal mean. The Nonlocal Mean is used to filter the noise from the image. It will produce visually good image with high PSNR value. Also produce excellent result and better output compare with other existing methods. The analysis result of different filtering methods is shown in the Fig.2. And then the Compare the PSNR value of various filtering methods on Lena image is shown in the Table I.

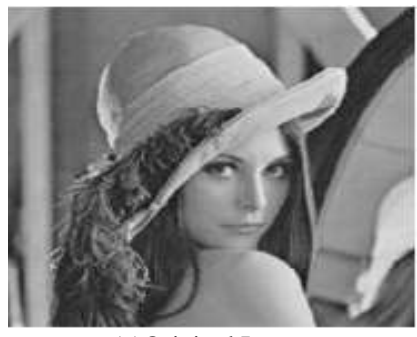

(a)Original Image

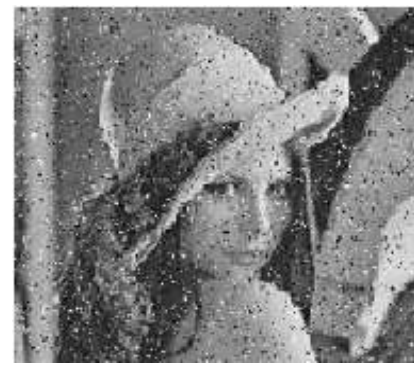

(b)CWMF 


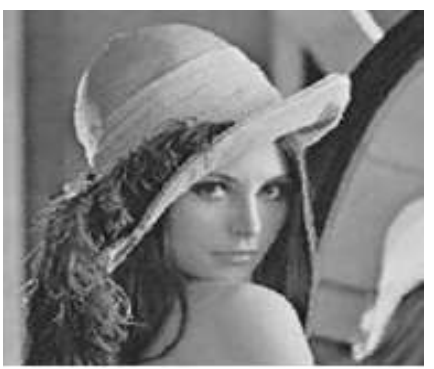

(c)AMF

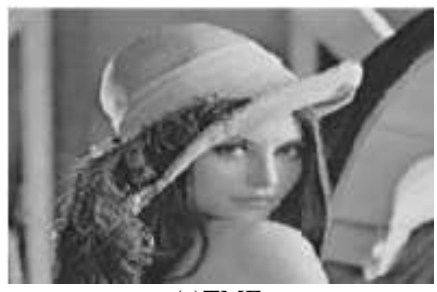

(e)TMF

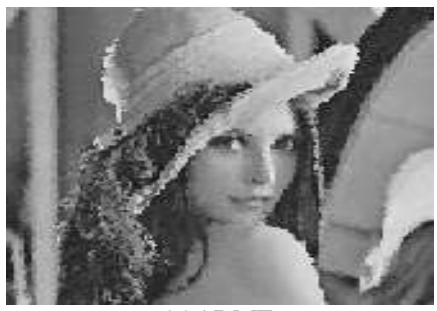

(g)ARMF

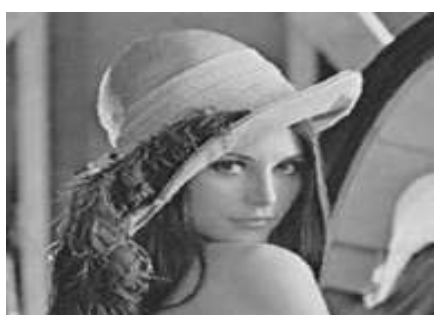

(i) DBAMF

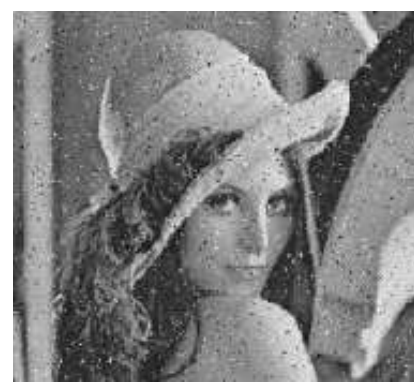

(d)PSMF

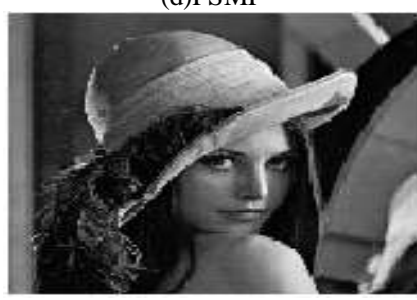

(f)MDB

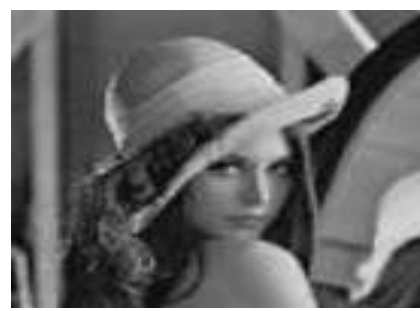

(h)ARE

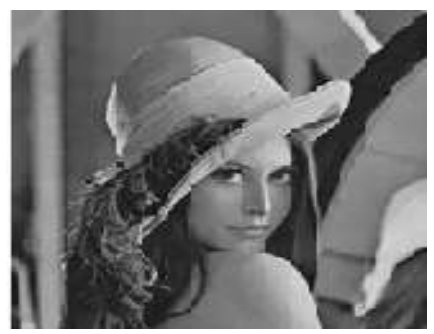

(j)ROR-NLM

Fig.2 Result of different filtering methods

TABLE I

COMPARE THE PSNR VALUE OF VARIOUS FILTERING METHODS ON LENA IMAGE

\begin{tabular}{|l|l|}
\hline Method & PSNR Value \\
\hline Center Weighed median filter & 25.68 \\
\hline Tri-state Median Filter & 27.26 \\
\hline Adaptive median filter & 36.73 \\
\hline Progressive Switching Median Filter & 29.69 \\
\hline Minmax Detector Based & 24.37 \\
\hline Adaptive Rank Order Filter & 32.37 \\
\hline Robust Estimation algorithm & 30.25 \\
\hline Decision based adaptive median filter & 28.76 \\
\hline Robust Outlyingness Ratio-NonLocal Mean filter & 32.45 \\
\hline
\end{tabular}

\section{Conclusion}

In this paper, we discussed different filtering techniques for denoising from the image. Further these techniques are comparing with each other. Finally conclude that among these techniques ROR-NLM is the good method to removing noise from the image. Even though it will produce high PSNR value it will produce high image quality by removing both Gaussian and impulse noise. 


\section{References}

[1] S.Kaisar, S. Rijwan, J.Al Mahmud, M. M. Rahman," Salt and Pepper Noise Detection and removal by Tolerance based Selective Arithmetic Mean Filtering Technique for image restoration", IJCSNS International Journal of Computer Science and Network Security, vol. 8,no .6, June 2008.

[2] G.Qiu “, An Improved Recursive MedianFiltering Scheme for Image Processing”, IEEE Trans. Image Processing, vol. 5, no. 4, april 1996.

[3] D. Brownrigg, "The weighted median filter," Commun. ACM, vol. 27, no. 8, pp. 807-818, Aug. 1984.

[4] Subg-Jea KO and Yong Hoon,"Center Weighted Median Filter and Their applications to image enhancement",IEEE Trans. Circuts and Systems, vol. 38,no. 9,Sept. 1991.

[5] H.Hawng and R.A.Haddad,"Adaptive Median Filter:Mew Algorithma and Result”,IEEE Trans.Image Processinf,vol. 4,no. 4,April 1995.

[6] T.Chen,Kai-Kuang Ma and Li-Hui Chen,"Tri-State Median Filter for Image Denoising”,IEEE Trans. Image Processing,vol. 8,no.12,Dec. 1999.

[7] Z.Wang and D.Zhang,"Progressive Switching Median Filter for the Removal of Impulse noise from Highly Corrupted Images", IEEE Trans. Digital Signal Processing vol. 46, no. 1 January 1999.

[8] X.Xu and E.L.Mller,"Adaptive Two Pass Median Filter to Remove Impluse Noise",IEEE-ICIP 2002.

[9] Xiaoyin Xu,E.L.Miller,D.Chen and M.sarhadi,"Adaptive Two-Pass Rank Order Filter to Remove Impulse Noise in Highly Corrupted Images”,IEEE Trans. Image Processing,vol. 13,no. 2,Feb. 2004.

[10] S. K. Satpathy, S. Panda, K. K. Nagwanshi and C. Ardil," Image Restoration in Non-Linear Filtering Domain using MDB approach", International Journal of Information and Communication Engineering 2010.

[11] V.R.Vijaykumar, P.T.Vanathi, P.Kanagasabapathy and D.Ebenezer, "High Density Impulse Noise Removal Using Robust Estimation Based Filter", IAENG International Journal of Computer Science, Aug. 2008.

[12] V.Jayaraj, D.Ebenezer, K.Aiswarya,"High Density Salt and Pepper Noise Removal in Images using Improved Adaptive Statistics Estimation Filter",IJCSNS International Journal of Computer Science and Network Security, vol. 9,no. 11, Nov. 2009.

[13] Bo Xiong and Zhouping Yin, "A Universal Denoising Framework with a New Impulse Detector and Nonlocal Means”, IEEE Trans. Image Processing, vol. 21, no. 4, April 2012. 